\title{
Centrin Phosphorylation and Localization Dynamics during the Centriole Cycle
}

Trace A. Christensen ${ }^{1}$, and Jeffrey L. Salisbury ${ }^{1}$

1. Department of Biochemistry and Molecular Biology, Mayo Clinic, Rochester, MN 55905

Centrin was first discovered as a major component of basal body-associated contractile flagellar roots in the algae, and subsequently was found to be a common protein of centrosomes [1-3]. Four centrin genes are expressed in mammals: Cetn-1 through Cetn-4 [4-7]. Centrin-2 and -3 are centriole proteins and recombinant GFP-centrin-2 and centrin-3 localize to centrioles and also to the pericentriolar material that surrounds the centrioles $[2,3,8,9]$.

Centrin is highly conserved in sequence and shows several striking structural features outside the four calcium-binding EF-hand domains. The carboxy-terminal half of centrin is highly conserved, and the short carboxy-terminal sequence - ${ }_{167} \mathrm{KKTSLY}_{172}$ - is conserved among centrins associated with centrioles and basal bodies. This sequence is missing in human centrin-3 and in centrins of lower eukaryotes lacking centrioles or in centrins that localize to cellular structures other than centrioles. Three putative Mps1 phosphorylation sites that augment the canonical centriole assembly pathway have been identified on centrin-2 [10]. Earlier, phosphorylation of centrin-2 at a distinct carboxy-terminal phosphorylation site $\left(-\mathrm{KKTS}^{170 \mathrm{P}} \mathrm{LY}\right)$ by protein kinase A was shown to promote centriole separation [11]. Finally, abnormal centrin phosphorylation S170 is observed in human breast tumors that have aberrant centriole numbers [12], suggesting that phosphorylation of centrin must be tightly regulated.

Here, we will present observations that show that in a mitogen-synchronized cell cycle, centrin abundance increases progressively and becomes phosphorylated at residue S170 prior to centriole duplication and DNA synthesis. Phospho-S170-centrin is asymmetrically localized to one of the preexisting centrioles at about the time that primary cilia are lost. The appearance of phospho-S170-centrin is temporally coincident with recruitment of robust SAS-6 staining to both pre-existing centrioles. A second peak of centrin S170 phosphorylation is seen at the onset of mitosis, preceding the late-mitotic ablation of centrin protein. Cells expressing conditional mutations at $S 170\left(S>A_{170}\right.$ and $\left.S>D_{170}\right)$ in centrin-2 show altered frequency of primary cilia and a unique pattern of PCM-satellite material reminiscent of failed centriole assembly.

The timing and localization of centrin phosphorylation and the opposing effects of conditional centrin mutations engineered to either block or mimic centrin phosphorylation suggest the intriguing possibility that centrin harbors a carboxy-terminal phosphorylation-sensitive alteration that corresponds to key events in the centriole cycle.

\section{References}

1. JL Salisbury, et al., Striated flagellar roots: isolation and partial characterization of a calciummodulated contractile organelle. J Cell Biol, 99(1984) p. 962-70.

2. AT Baron, et al., Centrin is a component of the pericentriolar lattice. Biol Cell, 76(1992) p. 383-8.

3. JL Salisbury, et al., Centrin-2 is required for centriole duplication in mammalian cells. Curr Biol, 12(2002) p. 1287-92. 
4. B Huang, et al., Molecular cloning of cDNA for caltractin, a basal body-associated Ca2+- binding protein: homology in its protein sequence with calmodulin and the yeast CDC31 gene product. J Cell Biol, 107(1988) p. 133-40.

5. R Errabolu, et al., Cloning of a cDNA encoding human centrin, an EF-hand protein of centrosomes and mitotic spindle poles. J Cell Sci, 107(1994) p. 9-16.

6. PE Hart and SM Wolniak, Molecular cloning of a centrin homolog from Marsilea vestita and evidence for its translational control during spermiogenesis. Biochem Cell Biol, 77(1999) p. 101-8.

7. S Middendorp, et al., Identification of a new mammalian centrin gene, more closely related to Saccharomyces cerevisiae CDC31 gene. Proc Natl Acad Sci U S A, 94(1997) p. 9141-6.

8. J Laoukili, et al., Differential expression and cellular distribution of centrin isoforms during human ciliated cell differentiation in vitro. J Cell Sci, 113(2000) p. 1355-64.

9. A Paoletti, et al., Most of centrin in animal cells is not centrosome-associated and centrosomal centrin is confined to the distal lumen of centrioles. J Cell Sci, 109(1996) p. 3089-102.

10. CH Yang, et al., Mps1 phosphorylation sites regulate the function of centrin 2 in centriole assembly. Mol Biol Cell, 21(2010) p. 4361-72.

11. W Lutz, et al., Phosphorylation of Centrin during the Cell Cycle and Its Role in Centriole Separation Preceding Centrosome Duplication. J. Biol. Chem., 276(2001) p. 20774-20780.

12. WL Lingle, et al., Centrosome hypertrophy in human breast tumors: implications for genomic stability and cell polarity. Proc Natl Acad Sci U S A, 95(1998) p. 2950-5.
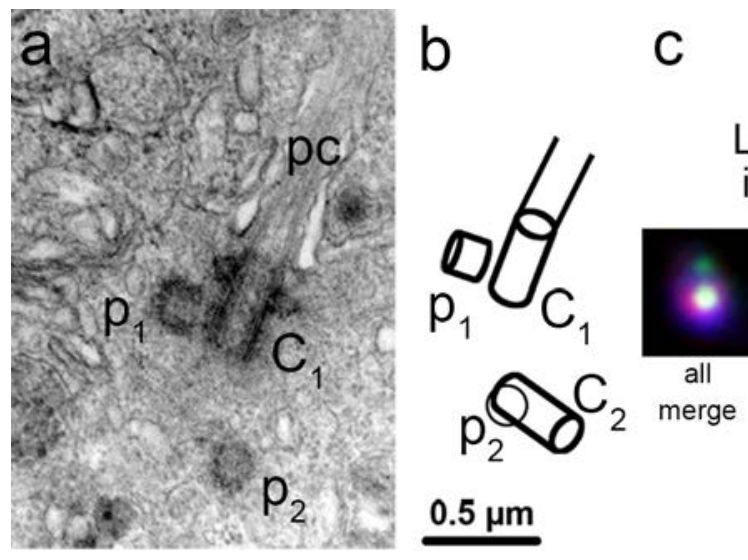

Localization of centrin in a pair of centrioles.

$0.5 \mu \mathrm{m}$

Figure Legend. a) Electron micrograph through a centrosome showing a primary cilium (pc), its centriole $\left(\mathrm{C}_{1}\right)$, and two procentrioles $\left(\mathrm{p}_{1}\right.$ and $\left.\mathrm{p}_{2}\right)$. b) Diagram illustrating the essential features shown in (a) with additional information from adjacent serial sections. c) Fluorescence microscopic localization of centrin in a pair of centrioles, showing a merged image of all channels, GFP-centrin2, phosphor-centrin at the carboxy-terminal sequence $\mathrm{KKTS}^{\mathrm{P}} \mathrm{LY}$, total centrin, and a model image with each of the channels adjusted to saturation. 